\title{
La investigación en Psicología de la Salud en Cuba: experiencias y potencialidades
}

\author{
Francisco Morales Calatayud \\ Universidad de Ciencias Médicas de La Habana
}

\begin{abstract}
Resumen
El artículo está dedicado al tema de la investigación en psicología en Cuba, con énfasis en el campo de la psicología de la salud. El propósito central está dirigido a compartir experiencias de su desarrollo y mostrar una aproximación a la situación actual, lo que puede ser de utilidad para explorar vías de cooperación en problemas de interés común. El tema es enmarcado en el contexto histórico-social y de política científica así como en el del desarrollo de la psicología en el país, con especial referencia a los últimos 50 años; se ofrece información sobre producción científica, eventos y temas en desarrollo en varias instituciones en el último decenio.
\end{abstract}

Palabras clave: Investigación en psicología; psicología de la salud; Cuba.

\begin{abstract}
Research in Health Psychology in Cuba: experiences and potencialities. The paper refers to psychological research in Cuba, and focuses on the field of health psychology. Main purpose is addressed to share experiences and show an approach to today situation as useful information in order to explore potential cooperation ways in common interest problems. Historic, social and scientific framework is described; summarized information about psychology in the last 50 years is offered, as well as references to some scientific meetings, research themes, scientific production, universities and institutes as a sample of last decade development.
\end{abstract}

Keywords: Research in psychology; health psychology; Cuba.

\section{Resumo}

Pesquisa em Psicologia da Saúde em Cuba: experiências e potencialidades. Este artigo dedica-se ao tema da investigação em psicologia em Cuba, com ênfase no campo da psicologia da saúde. Seu objetivo central é compartilhar as experiências de seu desenvolvimento e mostrar uma aproximação com a situação atual, o que pode ser útil na construção de estratégias de cooperação com relação a problemas de interesse comum. O tema é enquadrado no contexto histórico-social da política científica, assim como no desenvolvimento da psicologia no país, com especial referência aos últimos 50 anos. Além disso, são apresentadas informações sobre a produção científica, eventos e temas em desenvolvimento em várias instituições no último decênio.

Palavras-chave: Pesquisa em psicologia; psicologia da saúde; Cuba.

$\mathrm{E}$ ste trabajo está dedicado al tema de la investigación en psicología en Cuba, con énfasis en psicología de la salud. Su propósito central está dirigido a compartir experiencias de su desarrollo y mostrar una aproximación a la situación actual, lo que puede ser de utilidad para explorar vías de cooperación en problemas de interés común. Ha sido redactado para su presentación en la Mesa Redonda "Caminos de desarrollo científico e intercambio latinoamericano en psicología", en el III Encuentro Latinoamericano de Intercambio Universitario en Psicología", celebrado en Fortaleza, Brasil, en junio de 2010, esos encuentros constituyen una iniciativa muy valiosa para favorecer el conocimiento mutuo, algo que tanto necesitamos en América Latina.
El desarrollo de cualquier campo de la ciencia y la innovación tecnológica, se da en el marco de las relaciones sociales y está atravesado tanto por condiciones materiales, demandas utilitarias y disponibilidad de recursos como por circunstancias ideológicas, sociales, políticas, institucionales, grupales y hasta individuales. La producción de nuevos conocimientos, su difusión y aplicación en la práctica, está en permanente interacción con tales (y eventualmente otras) condiciones y circunstancias. La investigación, que es una actividad central dentro de la ciencia, está en permanente cambio y expresa claramente esa interacción; la investigación en psicología, disciplina en franca construcción que se ocupa del sensible tema de la esencialidad de lo humano, debe ser entendida en esa perspectiva. 
Es por eso que en este artículo habrá referencias a algunos aspectos del contexto histórico, del desarrollo en general de la ciencia y de la psicología en particular en el país; también se mostrarán referencias a experiencias e incluso a instituciones concretas, como un medio para establecer vínculos potenciales. Se previene al lector de que el tema no queda agotado, está apenas esbozado, refleja la perspectiva personal del autor y de que la información sobre áreas concretas es incompleta y resumida, se expone aquí sólo como una muestra; el énfasis en psicología de la salud se deriva del hecho de que ese es el campo donde se ha desarrollado la experiencia del autor. Se espera que en el futuro otros temas puedan mostrar visiones más amplias.

Cuba es un país insular situado a la entrada del Golfo de México, con una superficie de 110, $860 \mathrm{~km} 2$ y una población a fines de 2008, de 11'236, 099 habitantes (Oficina Nacional de Estadísticas, 2009). Es el mayor país insular de la región de América Latina y el Caribe, pero es realmente un país pequeño, sobre todo si se le compara con otros países de la región como Brasil, Argentina o México o con el cercano vecino, Estados Unidos. La población está formada fundamentalmente por descendientes de españoles y africanos, la convivencia y mestizaje de estos grupos durante siglos ha tenido un importante impacto en la cultura y la identidad del cubano contemporáneo.

\section{Desarrollo histórico de la Psicología}

Cuba tiene mucho en común con otros países latinoamericanos pero tiene también algunas peculiaridades. Tanto lo común como lo peculiar pueden resultar de interés, como contexto, en la comprensión del desarrollo de la psicología y la investigación en esta disciplina.

Fue la última de las colonias españolas en América. En 1902, al establecerse la república, los Estados Unidos impusieron limitaciones legales a la soberanía, también se apropiaron de los elementos fundamentales de la economía y reforzaron el proceso de penetración de la vida política y social, la educación se basó en modelos norteamericanos bajo la influencia del pragmatismo.

En este ámbito, de inicios del siglo XX, mientras en Estados Unidos y Europa se consolidaba la emergencia académica de la psicología, en Cuba, en el marco de las reformas educativas, se inicia la enseñanza de contenidos de psicología general en la facultad de Filosofía y Letras, influida por el conductismo norteamericano y en una etapa posterior por algunas corrientes psicoanalíticas; en la Facultad de Pedagogía se enseñaba psicología pedagógica en sus diversas ramas con una marcada influencia de la pedagogía norteamericana (Dewey entre otros) y de autores europeos como Claparéde y Binet (Rodríguez, 1990).

En los siguientes decenios, hasta el de 1950, se desarrolló la práctica de la psicología por egresados de profesiones afines o personas formadas en el exterior, fundamentalmente en tareas de evaluación psicológica, mediante el uso de tests aplicados a alumnos de escuelas privadas, pacientes de algunos consultorios de psiquiatría y en tareas de selección de personal en algunas empresas. Otra aplicación de la psicología estuvo dirigida al diseño y evaluación de campañas de propaganda comercial.

En ese periodo la investigación en psicología fue muy escasa. Hacia ese decenio, el de 1950, se realizaron algunos estudios de carácter crítico en relación con el uso indiscriminado de los tests y para la adaptación y validación de algunas pruebas por parte de valiosos investigadores pioneros. Un gran acontecimiento político, el triunfo de la Revolución en 1959, marcó un nuevo hito. En esta ocasión, según De la Torre (2006) la psicología experimentó un momento de ruptura que influyó enormemente en el curso que esta disciplina tendría en los próximos decenios.

Este hecho significó un cambio muy profundo en la vida de Cuba, tanto en las relaciones económicas como en las sociales. En este sentido, se abrieron amplias posibilidades para el acceso a empleos, educación, servicios de salud, deportes, recreación, mejores condiciones materiales de vida y participación social para amplios segmentos de la población hasta entonces preteridos. Al mismo tiempo, surgieron tensiones derivadas de la confrontación de clases y de la hostilidad desde el exterior, también otras tensiones que, aunque con sentido positivo, se derivaban de los importantes cambios en la vida de muchas personas: situaciones tales como la realización de nuevas tareas sociales y laborales, separaciones familiares por motivo de estudios, incorporación de las mujeres al trabajo, participación en tareas comunitarias y políticas, entre otras.

En el dominio de las subjetividades individuales en general, los proyectos de vida, las representaciones sociales, los valores, el desempeño de roles y las relaciones intrafamiliares e interpersonales, entre otras categorías psicológicas y sociales que podrían ser tomadas en cuenta, estaban ocurriendo en muy poco tiempo cambios de gran magnitud en la vida de muchas personas, como quizás nunca antes había ocurrido en la historia del país, fenómeno que requería interpretación y atención desde la psicología.

La Reforma Universitaria fue el marco apropiado para que en 1961 y 1962 surgieran las primeras escuelas de psicología en las universidades de Las Villas y La Habana, respectivamente (entonces sólo había 3 universidades en el país), hecho sobre el que hay coincidencia de opiniones en el sentido de que fue de gran importancia para el desarrollo posterior de la psicología en el país (De la Torre, 2006; Gónzález-Rey, 1995; Morales, 1997).

Se puede decir que la psicología, como desempeño profesional y como ciencia se hizo, progresivamente, parte del proyecto global de la sociedad, lo que no se expresó nunca en una declaración o documento oficial formal, resolución, decreto o ley de gobierno específica al respecto, sino que fue el resultado de un proceso de construcción paso a paso en el que intervinieron muchos factores, por ejemplo: la política social llevada a la práctica real; los programas de trabajo de sectores tan importantes como la educación y la salud, entre otros; la creación de espacios para la aplicación de la psicología en las más diversas instituciones; la vinculación con la realidad social de profesores y estudiantes de las universidades; las políticas de desarrollo académico y de ciencia e innovación tecnológica; el apoyo a la formación de postgrado; la motivación y creatividad de los propios psicólogos, muchos de los cuales, aún muy jóvenes asumieron tareas de alto compromiso social; la amplia disponibilidad de puestos de trabajo para estos; y la aceptación por parte de un pueblo cada vez más culto, de las potencialidades de la psicología para ayudar a resolver problemas de la vida 
cotidiana.

En este caso, se puede decir que es imposible separar el desarrollo de la psicología en los últimos cincuenta años, sus momentos de avance y de dificultades, sus aportes y sus limitaciones, del desarrollo de los procesos económicos, políticos y sociales generales en los que ese desarrollo se integra.

El centro de este trabajo no es la historia de la psicología en Cuba, esta primera parte sólo pretende servir de antecedente para que se comprenda mejor la parte dedicada a la situación actual en cuanto a investigación. Pero se puede ofrecer una información muy sintética siguiendo una periodización por decenios, como ha propuesto De la Torre (2006), los interesados pueden leer los trabajos más detallados que se citan en las referencias bibliográficas y otros sobre el tema, tanto sobre esta etapa como sobre las anteriores (Calviño, 2008; Escuela de Psicología, Universidad de La Habana, 1964; GonzálezRey, 1995; Guevara, 1984); aún queda mucho por investigar, sistematizar y publicar sobre este asunto. A continuación aparece dicha síntesis, con menciones muy escuetas a los hechos de contexto y en la psicología.

1960-1969. Se produce el comienzo de la formación profesional de psicología en las universidades, se observa un eclecticismo "saludable" en lo teórico (González-Rey, 1995), esto es, la utilización de varias perspectivas o abordajes tanto en el trabajo profesional como académico. Se desarrollan investigaciones por equipos de profesores y estudiantes de las universidades en problemas de interés en escuelas, comunidades, zonas agrícolas, industrias, barrios marginales. El Departamento de Psicología del Ministerio de Industrias es creado por el Comandante Ernesto Che Guevara, el primero de su tipo en una institución del gobierno central, se inician allí los estudios en temas de lo que hoy denominamos psicología organizacional (Arenas, González, \& Pérez, 1998; De la Torre, 2006).

Los primeros psicólogos egresados se insertan en diferentes instituciones. Ocurre el inicio de la integración de la psicología en el sistema de salud, priorizando el trabajo en la atención primaria y en las comunidades; surge el concepto de psicología de la salud; aparecen los primeros estudios de psicología institucional en ese sector así como sobre temas psicológicos en salud reproductiva y materno-infantil, nutrición, salud de los escolares, entre otros temas; comienza la integración de la psicología en la formación de pregrado de profesionales de la salud (García, 1980, 1981; Morales, 1997, 1999; Morales \& Ruíz, 1981).

1970-1979. Se extiende la práctica profesional a diversos sectores, la búsqueda teórica se orienta hacia la psicología de base marxista, con la tendencia en algunos a asimilar acríticamente modelos imperantes en países socialistas europeos (De la Torre, 2006). Se produce la franca emergencia de la psicología de la salud en todo el país, con diversidad de influencias teóricas y amplia creatividad; se desarrollan investigaciones en salud de la comunidad, enfermedades crónicas, discapacidades, salud reproductiva y materno-infantil y salud de los trabajadores, también investigaciones en psicología del desarrollo y educación especial, entre otros temas de interés social (Morales, 1997).

Se crean departamentos de psicología en los 12 institutos de investigación del sector salud, los que aportan resultados significativos en muy breve tiempo, tales como: adaptación y creación de instrumentos de evaluación psicológica para su uso en investigaciones sobre enfermedades crónicas no transmisibles y procedimientos para el trabajo de atención integral a las personas con estas enfermedades; inclusión de diversas variables psicológicas en investigaciones sobre enfermedades cardiovasculares, diabetes y otras endocrinopatías, trastornos gastrointestinales y otros, así como sobre salud de los trabajadores, de los escolares y nutrición, entre otras; se introducen contenidos de psicología en la formación especializada de los médicos que se forman en esos institutos (García, 1981; Morales \& Ruíz, 1981). En el sector de la educación también se fortalece la presencia profesional y científica de la psicología, con énfasis notable en la educación inicial y preescolar. Integración de psicólogos al desarrollo del deporte de alto rendimiento.

Surge en 1974 la Sociedad Cubana de Psicología de la Salud (García, 1980, 1981), posiblemente la primera sociedad científica con esa denominación en el mundo, fue establecida oficial y públicamente 4 años antes que las fechas que se señalan por Stone (1991) para la creación de la División de Psicología de la Salud de la American Psychological Association y 6 años antes que el grupo de trabajo sobre psicología de la salud de la Sociedad Interamericana de Psicología. Se realizan importantes congresos nacionales en el sector de la salud, como los congresos nacionales de psicología de la salud de 1975 y 1979, (este último con importantes participación de psicólogos latinoamericanos, norteamericanos y españoles). Jóvenes profesores realizan doctorados en el exterior (De la Torre, 2006).

1980-1989. Amplia presencia de la psicología profesional en diversos ámbitos, aumento del reconocimiento social y del nivel científico, incorporación de nuevos doctores; desarrollos teóricos propios; estudios orientados a la subjetividad, niveles de regulación de la personalidad, proyectos de vida, psicología educativa, motivación y procesos cognitivos, identidad del cubano, entre otros temas (De la Torre, 2006). Fortalecimiento de la psicología de salud tanto en su trabajo práctico y de investigación con comunidades como en su vinculación con la introducción de nuevas tecnologías de salud (terapia intensiva, fertilización in Vitro, trasplante de órganos, asesoramiento genético, etc.) y su inclusión en la formación posgraduada de médicos (Ministerio de Salud Pública de la República de Cuba, 1987; Morales, 1999).

Asimismo en este decenio, se produce la revitalización de la Sociedad de Psicólogos de Cuba y de la Revista Cubana de Psicología. Realización de importantes congresos internacionales en el país (como el de Psicología en la Comunidad en 1980, el Primer Congreso Internacional de Psicología de la Salud en 1984, el Interamericano de la S.I.P. en 1987 y Psicoanálisis y Marxismo en 1986 y 1988), aumento del intercambio y reconocimiento internacional. Es una etapa de auge en una época de importantes realizaciones globales de la sociedad cubana.

1990-1999. La psicología recibe el impacto de la crisis derivada de las afectaciones producidas a la economía y la sociedad cubana en general tras la caída del campo socialista y la 
intensificación del bloqueo; las dificultades de la vida cotidiana, el deterioro de las condiciones de trabajo y de transporte, entre otras, afectan la estabilidad emocional y los proyectos de vida de muchas personas, se tensionan los sistemas familiares. Se incrementa la demanda de atención psicológica; al mismo tiempo faltan recursos para investigaciones, eventos científicos, bibliografía, equipos; algunos psicólogos cambian sus tareas profesionales por otras; las demandas sociales apuntan más a las acciones prácticas que a lo investigativo (De la Torre, 2006).

Se mantiene, no sin afectaciones y con gran esfuerzo, el desarrollo de la psicología de la salud con énfasis en la atención primaria, así como de la psicología en la educación en general, en el deporte; se realizan investigaciones aplicadas en problemas tales como funcionamiento familiar, estrés y sistemas de apoyo, alcoholismo, drogas, infecciones de transmisión sexual, entre otros. Aumenta el interés del público por los temas de psicología, surgen nuevos espacios en medios de comunicación. No obstante las dificultades, se crea una nueva facultad de psicología en Santiago de Cuba, se inicia el programa de maestría en psicología de la salud (Louro, Casal, Martín, Hernández, \& Aguilar, 2009) y otros programas de posgrado encaminados a la formación de investigadores, así como el programa de especialidad en psicología de la salud, más enfocado al desempeño profesional.

2000-2009. Aunque este decenio “está ahí mismo" y no hay suficiente distancia para apreciar los aspectos más relevantes del mismo, puede considerarse que se aprecia una recuperación en términos generales, $\mathrm{y}$ en términos más particulares, un notable énfasis en la formación de recursos humanos de psicología. Se extiende la formación de grado a todo el país a través del programa de las sedes universitarias municipales, los resultados de este programa son evaluados en la actualidad. Se desarrolla la carrera de psicología en las universidades especializadas en el campo de las ciencias médicas y de la salud, con fuerte vinculación con la práctica (Louro et al., 2009).

Se ponen en marcha nuevos programas de maestría, algunos iniciados en el decenio anterior reciben acreditación de excelencia, pero la formación de doctores avanza más lentamente. La investigación se fortalece, especialmente en el abordaje multidisciplinario de temas de gran interés social (en otra parte de este trabajo, en atención al objetivo del artículo, se ofrece información más específica sobre el asunto de la investigación y la producción científica en la actualidad, lo que refleja tendencias que ya se vienen dando desde este decenio). Se recupera la actividad de las sociedades científicas, la celebración de eventos, la publicación de libros.

Resumiendo el punto de vista del autor de este artículo sobre el tema del desarrollo histórico de la psicología en Cuba: refleja una vinculación muy estrecha con el devenir histórico del país. En los últimos 50 años ha prevalecido un fuerte compromiso social de la psicología a través de sus instituciones y de los psicólogos en su actuación ya sea profesional, docente o investigativa, así como una tendencia reconocible a la integración de la psicología a los más diversos campos de la vida del país, con rigor y creatividad. El principal motor de este desarrollo han sido las demandas surgidas de una práctica diversa e intensa y la permanente búsqueda de los mejores recursos del conocimiento para contribuir al mejoramiento humano. Las principales dificultades han estado vinculadas tanto a las limitaciones de recursos como a la adopción de enfoques estrechos, miméticos y eventualmente dogmáticos.

\section{Enfoques de política cientifica}

También las políticas científicas en Cuba han tenido una relación muy estrecha con el devenir histórico del país. A principios del de decenio de 1960 se imponía en el mundo la tendencia a la adopción de políticas científicas bajo la idea de la "ciencia como motor del desarrollo", la que se extendió hasta fines del decenio de 1970 (Saez \& García, 1989). De acuerdo a esta idea de política científica, los resultados de la ciencia conducen a la creación de nuevas tecnologías y éstas al desarrollo, como si este último fuera una consecuencia directa de lo anterior. Más adelante prevalecería en la base de las políticas científicas la idea de la ciencia orientada a la solución de necesidades específicas, por último, a partir del decenio de 1990 y hasta ahora, ha prevalecido la concepción de la ciencia como recurso estratégico.

En Cuba en esa época se dedicó una parte importante de los recursos al fomento de nuevas instituciones científicas, a la ampliación de las capacidades de formación de las universidades y a incentivar la participación de éstas en investigaciones aplicadas en asuntos concretos de interés social. En la psicología se corresponde con lo descrito en los decenios de 1960 y 1970.

La etapa siguiente, la de la ciencia enfocada a necesidades precisas, tuvo una vida muy breve en el país, ya que en el decenio de 1980 se lograron en Cuba importantes resultados de punta en campos de la ciencia y la innovación tecnológica tales como la biotecnología y las neurociencias, entre otros, que condujeron a situar al país entre los primeros en adoptar la concepción más amplia e integral de la ciencia como recurso estratégico y en organizar un sistema de ciencia e innovación tecnológica que se ha ido fortaleciendo paso a paso, con muchísimo esfuerzo, pero también con valiosos logros, aun en los durísimos años del decenio de 1990.

Vale decir que si bien dicho sistema surgió movido por los avances en los campos mencionados antes, su existencia y la concepción de ciencia que le subyace, ha favorecido en general a todos los sectores.

Algunas expresiones concretas del sistema pueden ser mencionadas para una aproximación a la comprensión del entorno cubano: por ejemplo, la permanente actualización de la proyección estratégica en ciencia e innovación tecnológica de sectores e instituciones, y dentro de estas, el establecimiento y actualización sistemática de las prioridades de investigación; la organización de programas nacionales y ramales (para determinados sectores) con prioridades definidas; la actividad normativa y evaluadora de un organismo rector a nivel nacional y territorial; la participación activa de las universidades tanto en la investigación como en la formación de investigadores; el fortalecimiento de la cooperación; el seguimiento de los proyectos desde su aprobación hasta la introducción y generalización en la práctica de los nuevos o mejorados conocimientos y productos, entre otros (Pino \& Quevedo, 2010). 
Por otro lado, no puede decirse que todos los sectores y campos de la ciencia se desarrollen con igual vitalidad. Los recursos son sumamente escasos por razones que ya han sido mencionadas y a pesar de los esfuerzos armonizadores no resulta posible siempre disponer de la base material para algunos proyectos, especialmente en áreas, como por ejemplo, algunas ciencias básicas, en las que se hace menos visible anticipadamente el posible impacto inmediato del nuevo conocimiento que se pretende obtener. La movilización de recursos externos se impone como una necesidad.

Resumiendo este punto: es difícil establecer y decir de forma sintética en qué medida y dirección la política científica del país ha impactado en la investigación en psicología, ya que esta se produce en diversos escenarios institucionales, tanto académicos como de la producción y los servicios. Esto es algo que tenemos que estudiar mejor, pero, nuevamente sobre la base de la apreciación global de este autor, se considera que la psicología se favorece de la prioridad dada a la ciencia y la innovación tecnológica en sectores donde está bien representada, como los de la educación general y superior y el de la salud. Sin embargo, aún es posible alcanzar mejores niveles de articulación en cuanto a complementación de proyectos y cooperación entre instituciones, cultura de proyectos y movilización de recursos externos.

\section{Formación de investigadores}

Los primeros investigadores de la psicología cubana, aquellos que hicieron las investigaciones pioneras sobre pruebas psicológicas en el decenio de 1950 y las primeras investigaciones sobre psicología social, educacional y clínica, en el decenio de 1960, se formaron de modo autodidacta y complementando su formación profesional con algunos cursos cortos en el exterior. A partir de ese decenio se abren las posibilidades para que algunos de los primeros egresados de la carrera, ahora jóvenes profesores, recibieran formación científica en países de Europa, como por ejemplo, Francia y Bélgica.

Después, desde mediados del decenio de 1970, el flujo de intercambio con los países entonces dentro del campo socialista favoreció que más de tres decenas de psicólogos recibieran formación doctoral en psicología hasta mediados del decenio de 1980. Esto fue un impulso notable en corto tiempo; para un país pequeño constituía una buena cantera que permitió establecer la formación de especialistas, masters y doctores en el país.

El programa doctoral vigente en la actualidad es sólo en la modalidad tutelar, con cuatro programas de especialidad: psicología general, psicología social y del trabajo, psicología educativa y del desarrollo y psicología clínica. En la actualidad se trabaja en el desarrollo de al menos un programa curricular colaborativo para este doctorado. En otros programas de doctorado, como los de Ciencias de la Salud, Ciencias Pedagógicas y Ciencias de la Educación se han formado otros psicólogos para la obtención de este grado.

A principios del decenio de 1990 surgieron los programas de maestría, orientados fundamentalmente a la formación de investigadores; entre los primeros estuvo el de Psicología de la Salud, en la Escuela Nacional de Salud Pública, el que ha tenido desde entonces un exitoso desarrollo y está acreditado como programa de excelencia por la exigente Junta de Acreditación Nacional. A mediados de esa década surgió un programa de especialización en psicología de la salud que también incluye formación en investigación (Louro et al., 2009).

Otros programas de maestría que surgieron en las universidades del campo de las ciencias de la salud desde esa época o antes, en temas interdisciplinarios, han contribuido a la formación como investigadores de aproximadamente un centenar de psicólogos, este es el caso de las maestrías en Salud Pública, Atención Primaria en Salud, Promoción y Educación para la Salud, Salud de los Trabajadores, Sexualidad, Longevidad Satisfactoria, Salud Materno Infantil, Psiquiatría Social y Psicología Clínica, entre otras. Aproximadamente 1 de cada 4 psicólogos del sector de la salud ha cursado un programa de maestría o especialidad, o lo está cursando en la actualidad.

En otras universidades, del mismo modo, se ha producido un desarrollo cada vez más amplio hacia los programas de maestría, por ejemplo, la Facultad de Psicología de la Universidad de La Habana desarrolla programas de Maestría en Psicología Clínica, Psicología Educativa, Psicología Organizacional, Psicología Social y Comunitaria, Procesos Directivos y Recursos Humanos y en Psicodrama y participa en la Maestría sobre Género de la Cátedra de la Mujer de esa universidad (R. Rojas, comunicación personal, 21 de mayo de 2010); la Universidad Central de Las Villas desarrolla otros programas, por ejemplo, el de la Maestría en Psicología Médica.

La formación de investigadores se ha extendido en número en los últimos 15 años, sin embargo, no hay una adecuada conexión entre el nivel de maestría y el de doctorado, algo que los programas curriculares en desarrollo buscan resolver.

\section{Producción Científica}

Antes de entrar en este tema, sería bueno volver a hablar de algunas características del país, que de alguna manera se reflejan en la producción científica en psicología.

Datos al cierre de 2008, muestran que Cuba tenía una esperanza de vida al nacer de 77,97 años, y que el 17\% de la población del país tiene 60 años y más, la tasa de mortalidad infantil era de 4,7 por cada mil nacidos vivos. La primera causa de muerte fue la de Enfermedades del corazón, seguida por Tumores malignos y Enfermedades cerebrovasculares. Más del $99 \%$ de los niños de 6 a 11 años asisten a la escuela primaria y más del 85\% del grupo de 11 a 17 años, a la enseñanza secundaria. Una de cuatro personas está vinculada a algún programa educativo (Oficina Nacional de Estadísticas, 2009). Es un país con muchas limitaciones económicas, pero con alta prioridad en la educación y en la salud. Más de la mitad de los psicólogos cubanos trabajan en esos sectores priorizados.

No es extraño entonces que temas relacionados con la salud y la educación aparezcan entre los más frecuentes en la producción científica de los psicólogos cubanos.

Esa producción está dispersa en presentaciones en diversas instituciones, eventos y revistas, una buena parte de éstas últimas, no son específicas de psicología pero publican en ellas los psicólogos, ya sea como autores principales o como miembros de colectivos multidisciplinarios.

Se presenta a continuación en examen resumido de algunas 
muestras de la producción científica presentada en congresos que pueden ser considerados representativos, ya que tienen alcance nacional, amplia participación y son organizados por sociedades científicas bien establecidas, rigurosas en la selección de los trabajos a presentar en tales eventos. La información ha sido obtenida de las memorias de esos eventos editadas en soporte digital rígido.

Congreso Hominis. Organizado por la Sociedad Cubana de Psicología, celebrado en 2005. De acuerdo al perfil de este evento, participaron mayoritariamente psicólogos del ámbito de las universidades. Hubo más de 250 presentaciones de autores cubanos, entre conferencias, talleres, ponencias teóricas, 1 de cada 4 de esas presentaciones informa el resultado de una investigación empírica en temas de psicología y realizada por psicólogos.

De ellos, aproximadamente la mitad están relacionados con el campo de la salud, por ejemplo: experiencias con técnicas de grupo en formación de promotores de salud; resultados de programas para prevención de enfermedades de transmisión sexual y de adicciones; descripción de manifestaciones psicológicas en enfermedades crónicas e intervenciones en las mismas; atención a pacientes en estadío terminal; y psicoterapia infantil. En este congreso destacan otros temas como sexualidad y género. Asimismo se presentan un gran número de trabajos teóricos, en particular sobre el enfoque histórico-cultural y sobre psicodrama, asuntos que tuvieron sesiones especiales.

Congreso Psicosalud 2008. Organizado por la Sociedad Cubana de Psicología de la Salud, la mayoría de participantes fueron psicólogos de este sector. En una revisión de 80 trabajos de autores cubanos que informan sobre investigaciones empíricas se observa que los temas que con más frecuencia aparecen son los siguientes:

Adulto mayor. Sistemas de apoyo familiar, cuidadores, comunicación, trabajo grupal para el fomento de la salud con estas personas, entre otros asuntos.

Manifestaciones psicológicas e intervenciones con personas con enfermedades crónicas. Hay trabajos en relación con el asma, el cáncer en general y de localizaciones específicas, como el de mama (comunicación de información, grupos de apoyo), en Alzheimer y epilepsia, entre otras enfermedades.

Sexualidad y salud reproductiva. Aparecen estudios en relación de la salud reproductiva en adolescentes y su promoción, prevención de enfermedades de transmisión sexual, apoyo familiar en el embarazo, entre otros.

Enseñanza de la psicología. En la formación de recursos humanos del campo de la salud y a psicólogos en universidades de este sector.

En cuanto a revistas, la presencia de informes de investigaciones de psicología está limitada por diversas razones, la principal es que la publicación de la Revista Cubana de Psicología quedó interrumpida a mediados de la última década, a pesar de los esfuerzos de sus editores; en la actualidad se trabaja en su recuperación como revista electrónica. Los psicólogos publican en otras revistas, entre ellas, las del sector salud. Por ejemplo, en la Revista Cubana de Salud Pública (4 números al año) hay 18 informes de investigaciones de psicólogos en los volúmenes aparecidos de 2006 a 2009, dentro de un conjunto de 80 artículos con informes de diferentes áreas de la salud pública comprendidos en el apartado dedicado a investigaciones en esta publicación. Entre los temas que abordan esos artículos se encuentran estudios relacionados con: la caracterización psicosocial de cuidadores de adultos mayores con demencia, diseño y evaluación de programas psicoeducativos para los cuidadores; la percepción de riesgo del tabaquismo; la prevención del alcoholismo; el comportamiento de adherencia terapéutica en la hipertensión arterial; la caracterización psicosocial de las personas con diabetes tipo 2 y la evaluación de la satisfacción con la salud, entre otros temas. Otras revistas que acogen trabajos de psicología con regularidad son las revistas Cubana de Medicina General Integral y Cubana de Salud y Trabajo. La primera de estas acoge preferentemente trabajos de profesionales de las instituciones de atención primaria de la salud y es posible hallar allí asuntos que se abordan de manera conjunta desde la perspectiva y amplio espectro de asuntos que tratan las disciplinas que participan en la atención de la salud de las personas en la comunidad. En el periodo comprendido entre 2006 y 2009, en 16 números de esta revista aparecen 11 artículos que incluyen asuntos psicológicos dentro del conjunto de 93 publicados en la sección de "artículos originales". Los temas de los mismos se refieren a asuntos tales como deterioro cognitivo en personas de edad avanzada; satisfacción con la vida en mujeres de edad mediana; atención al estrés familiar; repercusión psicosocial del paso de un huracán; comportamiento sexual de adolescentes; variables psicosociales en la estratificación de la salud familiar; también en esta revista hay trabajos sobre adherencia terapéutica en personas con hipertensión arterial y sobre el impacto psicosocial del síndrome demencial en cuidadores. La segunda revista (Salud y Trabajo) con 7 números en el mismo período, incluye 72 trabajos en conjunto, dentro de los cuales hay 9 sobre temas psicológicos, tales como validación de instrumentos para el diagnóstico del mobbing; estrés psicosocial laboral en jóvenes embarazadas; variables de personalidad y síndrome de desgaste psicológico en el trabajo; evaluación conductual y estado de salud en trabajadores de salas de operaciones, entre otros. Otras revistas del campo de la salud en Cuba pueden mostrar trabajos de psicología como expresión de la integración de los psicólogos en diversas áreas. Estas revistas pueden encontrarse en las bases de datos de sitios como SciELO y otros.

En revistas internacionales de psicología, como la Interamericana, son poco frecuentes los trabajos de autores cubanos, lo que puede ser expresión de una insuficiente cultura de publicación en el exterior.

\section{Algunas áreas de interés en investigación en psi- cología de la salud en Cuba hoy}

Aquí se mencionan solamente algunas áreas e instituciones, a modo de ejemplo, tanto de la actividad en el marco universitario como de algunas instituciones, especialmente del sector salud, en estas últimas se incluyen ejemplos tanto del área de la atención 
a personas enfermas como del área de salud pública.

Universidades del campo de salud. Como un resultado de la amplia política de formación de recursos humanos para el sector salud, en los últimos 35 años se creado progresivamente una red de universidades que abarca todo el país (una por provincia, 14), las que cuentan con la carrera de psicología (mención salud), en las que se enseña psicología en el pregrado de todas las demás carreras y en la mayor parte de los programas de formación de postgrado y que tienen, entre otras fortalezas, la de servir de plataforma a la investigación en ese campo y para la coordinación del trabajo de ciencia e innovación tecnológica en salud en todo el país. Dentro de este, existen prioridades expresadas en programas "ramales" (de todo el sector) y "territoriales" (en cada provincia).

Esta red constituye un importante marco para la investigación en psicología de la salud, los psicólogos están bien representados en el trabajo académico de esas instituciones y las instituciones docente-asistenciales-investigativas que les están adscriptas, lo que ha favorecido mucho el trabajo de investigación en problemas que requieren ser abordados de conjunto con otras disciplinas de este campo y la producción de conocimientos relacionados con problemas de salud valorados como de interés prioritario.

Por ejemplo, en la Universidad de Ciencias Médicas de La Habana, entre las áreas con más actividad están las de aspectos psicológicos relacionados con los siguientes asuntos: Promoción de Salud en la Comunidad; Bienestar Subjetivo y Calidad de la Vida en personas con enfermedades crónicas; Estrés en el riesgo de enfermar; Adulto Mayor; Familia y Salud; Salud reproductiva y materno-infantil; Prevención de adicciones; Violencia Intrafamiliar (Molina, 2004); SIDA y otras enfermedades de transmisión sexual; Psicoterapia e Hipnosis; Psicología en la formación de recursos humanos para la salud; Formación de psicólogos orientados al sector salud; Integración de la psicología en los servicios de salud (A. I. Martínez-Portuondo, comunicación personal, 10 de mayo de 2010). Cada una de estas universidades tiene sus propios desarrollos y fortalezas, un estudio de "investigación sobre la investigación" que está en fase de proyecto en el momento actual, permitirá más adelante disponer de información más detallada sobre el conjunto de este trabajo científico y sobre sus particularidades en cada una de estas universidades.

Escuela Nacional de Salud Pública. Centro de coordinación de estudios e investigación en este campo, tiene una maestría de excelencia en Psicología de al Salud. Áreas de más actividad: Calidad de Vida, Bienestar Subjetivo y Salud; Situación de Salud de la Familia; Evaluación y Perfeccionamiento de las Organizaciones de Salud; Promoción de la Conducta Salutogénica y Prevención del Riesgo Psicológico de Enfermar (Escuela Nacional de Salud Pública, 2010).

Facultad de Psicología de la Universidad de La Habana. Centro de referencia en la formación de recursos humanos en psicología en el país, las áreas de más actividad son las siguientes: Género; Adultos Mayores; Aprendizaje y Desarrollo Humano; Familia, Pareja y Sexualidad; Psicología Especial y de las Personas con Discapacidades; Salud y Calidad de Vida; Psicoterapia; Salud Comunitaria; Procesos de Dirección y Liderazgo (R. Rojas, comunicación personal, 21 de mayo de 2010).

Instituto Nacional de Oncología y Radiobiología. En este Instituto comenzó el desarrollo de la psicooncología desde 1990, el departamento de Psicología mantiene un trabajo sostenido de integración asistencial, docente e investigativa en general en ese campo en asuntos tales como: Manifestaciones psicológicas, Sistemas de Apoyo, Comunicación de Información, Manejo del dolor, Cuidados Paliativos, Oncopediatría, Burnout, Sufrimiento y Manejo de Esperanza; así como en Calidad de Vida, Prevención y Educación en Cáncer (una muestra de resultados de investigación de este centro se aprecia en Llantá et al., 2008) recientemente ha comenzado un programa en la comunidad en un centro de atención primaria, dirigido al control de sintomas y calidad de vida como indicador de impacto en pacientes de cáncer avanzado y en caracterización del duelo ( $\mathrm{J}$. Grau, comunicación personal, 4 de junio de 2010).

Instituto de Salud de los Trabajadores. Durante más de 35 años ha tenido una activa producción científica en psicología aplicada a la salud ocupaciónal (Estrés laboral y su relación con determinadas condiciones de salud así como con la dinámica familiar; Desgaste profesional; Ambientes laborales saludables; Psicotoxicidad, entre otros). Una información más amplia puede encontrarse en Almirall y Hernández (2010).

Departamento de Psicología del Centro Internacional de Restauración Neurológica. Ejemplo de vinculación de las neurociencias básicas y la psicología. Entre sus temas están el estudio de las Bases Neurales de la Cognición (en el marco del proyecto de Mapeo Cerebral Humano); Alteraciones Psicológicas en la enfermedad de Parkinson; Rehabilitación Cognitiva Computarizada; Cambios Cognitivos asociados al Trasplante de Células Madre (M. L. Bringas, comunicación personal, 19 de mayo de 2010).

Área de Psicología del Centro Latinoamericano de Medicina de Desastres. Área que se fortalece cada vez más, con amplia experiencia y resultados en diversidad de situaciones. Entre sus temas se encuentran: Preparación Comunitaria para la reducción de desastres; Riesgos y Vulnerabilidades Psicosociales para Desastres en comunidades cubanas; Repercusiones Psicosociales de Desastres; Desarrollo de Metodologías Psicológicas para la actuación en situaciones de Desastres (Lorenzo, 2006; A. Lorenzo, comunicación personal, 22 de mayo de 2010).

Existen otras áreas muy activas en la propia psicología de la salud y otros campos así como en centros académicos tales como las universidades Central y de Oriente y de otros sectores como la educación, el deporte, los estudios sociales, de organizaciones y la cultura. Una reseña más detallada sobrepasa los límites de este artículo.

Las potencialidades de desarrollo de la investigación en psicología de la salud en Cuba, puede considerarse, se basan fundamentalmente en a) la experiencia de más de cuatro décadas de amplia y activa participación de los psicólogos en 
instituciones académicas y de servicios del campo de la salud, en las que prevalece una política de integración de la psicología a los problemas de salud prioritarios; y b) la formación en competencias de investigación de un creciente número de psicólogos.

La búsqueda de oportunidades de colaboración con instituciones de otros países que comparte estas experiencias e intereses, así como la búsqueda de recursos externos para apoyar el trabajo de investigación requiere ser asumida como una prioridad, asimismo se hace necesario el desarrollo de la cultura de publicación en el exterior, de modo que se supere la baja visibilidad de la producción científica actual y que esto favorezca los vínculos con otras instituciones para el desarrollo de proyectos cooperados.

Es deseable que en el futuro se avance en estas direcciones y que la comunicación e intercambio con los psicólogos de Brasil ocupe un espacio importante en el interés de los psicólogos en el sector salud en Cuba.

\section{Agradecimientos}

El autor desea expresar su gratitud a todos cuantos brindaron su ayuda para este artículo, especialmente a Carolina de la Torre Molina, Marianela Morales Calatayud, Jorge Grau Ábalo, Pedro Almirall Hernández, Isabel Louro Bernal, María Luisa Bringas, Alexis Lorenzo Ruiz, Ada Casal Sosa, Jorge Díaz González, Carmen Regina Victoria García-Viniegras, Ana Ivonne Martínez Portuondo, Olga Infante Pedreira, Diego González Serra y Manuel Calviño Valdés-Fauly así como a Maria de Fátima Duarte Martins, de la Universidad de Pelotas, por su ayuda en la preparación de la versión en portugués para su presentación en Fortaleza.

\section{Referencias}

Almirall, P. J., \& Hernández, J. S. (2010). Investigación científica en el Instituto Nacional de los Trabajadores de Cuba (1998-2008): algunas reflexiones y proyecciones. Revista Cubana de Salud y Trabajo, 11(1), 59-70.

Arenas, P., González, J. C., \& Pérez, I. (1998). El desarrollo de la Psicología Organizacional en Cuba. La Habana: CIPS

Calviño, M. (2008). Breve ensayo sobre la psicología en Cuba. Revista Cubana de Psicología, (Número especial), 9-18.

De la Torre, C. (2006). Psychology in Cuba after 1959. History and Philosophy of Psychology, 8(1), 12-29.

Escuela de Psicología, Universidad de La Habana. (1964). Orientación, contenido y funciones de la psicología en Cuba. Revista Psicología y Educación, l(1), 90-95.

Escuela Nacional de Salud Pública. (2008). Programa de la Maestría de Psicología de la Salud. La Habana: Autor.

García, L. (1980). Psychology and health in Cuba. American Psychologist, 35(12), 1090-1095.

García, L. (1981). La psicología de la salud en Cuba: situación actual y perspectivas. In Pontificia Universidade Católica de São Paulo (Org.), Psicologia: reflexões sobre a prática da psicologia (Cadernos PUC 11, pp. 20-28). São Paulo: EDUC/Cortez.

González-Rey, F. (1995). La psicología en Cuba: apuntes para su historia. Revista Temas, 1, 69-76.

Guevara, J. (1984). El establecimiento y desarrollo de las ideas psicológicas en Cuba. Revista Cubana de Psicología, 1(1), 81-92.

Llantá, M. C., Pire, T., Grau, J., Vilau, L., Massip, C., Grau, R, \& Ortiz, R. (2008). Evaluación del sufrimiento en pacientes con quimioterapia del Instituto Cubano de Oncología y Radiobiología. Psicología y Salud, 18(2), 149-151.

Lorenzo, A. (2006). Reflexiones sobre la evolución del quehacer psicológico en el tema de emergencias y desastres. Análisis de la experiencia en Cuba. Cuadernos de Crisis, 2(5), 7-37.

Louro, I., Casal, A., Martín, L., Hernández, L., \& Aguilar, I. (2009). La formación de recursos humanos en psicología de la salud en Cuba a partir de 1959. Revista Cubana de Salud Pública, 35(1), 0-0. Recuperado de http://scielo. sld.cu/scielo.php?script=sci_issuetoc\&pid=0864346620090001\&lng=esn $\mathrm{rm}=$ iso\&tlng $=\mathrm{es}$

Ministerio de Salud Pública de la República de Cuba. (1987). Programa de Desarrollo 2000. Psicología de la Salud. La Habana: Ciencias Médicas.

Molina, M. C. (2004). La investigación psicosocial en la atención primaria de salud. La Habana: Interpsiquis.

Morales, F. (1997). La Psicología y los servicios de salud: experiencias de trabajo en Cuba. Buenos Aires: Oficina del Ciclo Básico Común/Universidad de Buenos Aires.

Morales, F. (1999). Introducción a la psicología de la salud. Buenos Aires: Paidós.

Morales, F., \& Ruíz, G. (1981). Temas relevantes en la investigación de aspectos psicosociales en salud. In Pontificia Universidade Católica de São Paulo (Org.), Psicologia: reflexões sobre a prática da psicologia (Cadernos PUC 11, pp. 35-40). São Paulo: EDUC/Cortez.

Oficina Nacional de Estadísticas. (2009). Anuario Estadístico de Cuba. La Habana: Autor.

Pino, L., \& Quevedo, V. (2010). Introducción a la innovación: el sistema cubano de ciencia e innovación tecnológica. In Colectivo de Autores (Org.), Curso de Innovación para el Desarrollo (pp. 2-19). La Habana: Academia.

Rodríguez, A. (1990). Transitando por la psicología. La Habana: Ciencias Sociales.

Saez T., \& Garcia, E. (1989). Ciencia y tecnología en Cuba. La Habana: Ciencias Sociales.

Stone, G. C. (1991). An international review of the emergence and development of health psychology. In M. A. Jansen \& J. Weinman (Orgs.), The international development of Health Psychology (pp. 3-17). Chur: Harwood Academic Publishers.

Francisco Morales Calatayud, doctor en Ciencias de la Salud en la Universidad de Ciencias Médicas de La Habana, es profesor Titular Consultante de Psicología en la Universidad de Ciencias Médicas de La Habana. Correspondencia: Calle Zapata y D, Plaza de la Revolución, La Habana 10400, Cuba. Correo electrónico: fmorales@infomed.sld.cu 